\title{
Bio-Inspired Polymersome Nanoreactors
}

\author{
Omar Rifaie-Grahama, Edward A. Apebende ${ }^{a}$, and Nico Bruns ${ }^{\star a b}$
}

\begin{abstract}
Two key concepts in living organisms are that biochemical reactions are sequestered into reaction compartments such as cells and organelles, and that many of the complex biological reaction cascades involve transient activation of reactions in response to external triggers. Here we review our efforts to implement these concepts into artificial nanoreactors. Block copolymer vesicles (polymersomes) for laccase-catalyzed oxidations as well as a generally applicable permeabilization method for polymersome membranes are highlighted. Moreover, polymersome nanoreactors that can be switched on by visible light and that immediately return to their off state in the dark are reviewed. These systems have the potential to create bio-inspired catalytic systems, e.g. to orchestrate reaction cascades.
\end{abstract}

Keywords: Amphiphilic block copolymers · Biocatalysis · Nanoreactors · Transient out-of-equilibrium systems - Vesicles

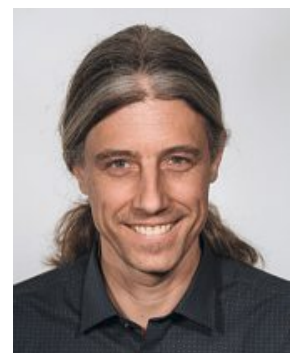

NicoBruns is a Professor of Macromolecular Chemistry at the University of Strathclyde, Glasgow. Before moving to Scotland in October 2018, he was Associate Professor at the Adolphe Merkle Institute of the University of Fribourg (Switzerland), funded by a Swiss National Science Foundation Professorship. He habilitated in chemistry from the University of Basel, was a postdoc in biotechnology at the University of California, Berkeley, and obtained his $\mathrm{PhD}$ in macromolecular chemistry from the University of Freiburg (Germany). Nico Bruns is a founding member and principal investigator of the National Center of Competence in Research (NCCR) Bio-Inspired Materials and coordinates the EU-funded Innovative Training Network Plant-Inspired Materials and Surfaces (PlaMatSu). His research interests include polymersome- and protein-based nanoreactors, biocatalytic atom transfer radical polymerizations, and force-responsive protein-polymer hybrid materials.

\section{Introduction}

A key concept of life is that biochemical reactions occur in defined reaction compartments. ${ }^{[1]}$ Every living organism can be regarded as a biochemical factory that is partitioned from the environment by a shell, such as skin or the cuticle of leaves. This shell has many functions, one being that it blocks certain substances from entering or leaving the interior, while modulating the transfer of other substances across it, often in response to specific stimuli. Every cell is a micron-scale reaction compartment that hosts biochemical machinery to sustain the function of the cell. Within eukaryotic cells, organelles provide yet another smaller kind of reaction compartment. These various levels of hierarchically organized reaction compartments allow for complex biochemical transformations to be achieved in a much more efficient way than if all reagents and biocatalysts would be mixed together. For example, reagents and substrates that are not compatible with each other are sequestered into different reaction compartments; conversely, the efficiency of reaction cascades can be enhanced by keeping a set of biocatalysts in close spatial proximity within one reaction compartment. Moreover, the control of transport of reagents, substrates, and products across the membranes of organelles and cells is a key element to enable temporal control over biochemical reactions, e.g. to turn them on and off, or to modulate the rate of certain reactions in the compartments by adjusting the rate at which substrates are transported across the cell membrane.

Another important concept in nature is that many of the processes in cells are transient, i.e. they happen as long as a certain stimulus is present, and stop and revert to their starting state as soon as this stimulus is withdrawn. ${ }^{[2]}$ For example, in photoreceptor cells, light triggers the cis-trans isomerization of retinal, which results in a cascade of reactions that ultimately lead to the sense of vision. If retinal would remain in its light-activated trans form, the photoreceptor cells would not be able to repeatedly sense light. Thus, an enzymatic reaction cascade converts the trans retinal back into its starting cis isomer. On a conceptual level, the two isomers can be considered in an equilibrium that shifts towards the trans retinal when irradiated with light, and shifts back (through enzymatic action) in the absence of the stimuli, i.e. in the dark. Here, we will review our efforts to impart both of these concepts, compartmentalization of reactions and transient out-of-equilibrium response to external stimuli, in bio-inspired polymer nanosystems.

\section{Nanoreactors}

While most reaction compartments in cells are of micron scale, artificial reaction compartments are often designed to have dimensions on the nanoscale. ${ }^{[3-7]}$ This size regime allows for good mass transport into and out of the reactors. A nanoreactor partitions a solvent-filled volume from the outside environment by shell or wall. Any kind of nanocapsule can be used for this purpose, including protein cages ${ }^{[3,8,9]}$ or self-assembled polymer structures such as vesicles ${ }^{[3-7]}$ or micelles. ${ }^{[10]}$ The key element to these nanoreactors is that the boundary to the outside environment is permeable for the substrates of the desired reactions that will occur within the confined space of the reactor. Moreover, it is desirable (but not a necessity) that the products of the reactions 
can also leave the nanoreactor. A common strategy to confine reactions into nanoreactors is to encapsulate catalysts in the nanoreactors. Often, these catalysts are enzymes, but examples of other kinds of catalysts have also been reported.

We and others have explored for many years the potential of polymersomes, i.e. vesicles that form by the self-assembly of amphiphilic block copolymers, as nanoreactors. ${ }^{[3-7]}$ Polymersomes consist of an inner aqueous compartment surrounded by a polymer membrane that resembles, in many aspects, the phospholipid membrane of cells and organelles. The hydrophobic blocks of the polymers form a hydrophobic leaflet that is flanked at each side by a hydrated layer of the hydrophilic blocks. Polymersomes allow access to reactions in an inner aqueous lumen that is sequestered from the aqueous bulk solution through a thin hydrophobic polymer layer with a thickness typically in the range of 6-20 nm. ${ }^{[1]}$ The reason for the popularity of polymersomes is that they are very stable capsules, especially in comparison to their lipid counterpart, the liposomes. ${ }^{[7]}$ Once assembled into the membrane of polymersomes, the block copolymers tend to remain there and do not diffuse into solution, so that loaded polymersomes can be stored for months without losing their structural integrity and their content. Moreover, polymersomes are chemically very versatile and can comprehend a wide variety of chemical motifs. It is also straightforward to encapsulate biomacromolecules, such as enzymes, into polymersomes upon their formation. Due to the size and hydrophilic nature of enzymes, they remain in the polymersomes even if these are made permeable for small organic molecules. Additionally, the polymersome shell represents a barrier that keeps protein-degrading enzymes, such as proteases, away from the encapsulated enzymes, thus protecting the latter against degradation and deactivation. This is especially important if the nanoreactors are going to be used in a biological context, such as artificial organelles. The impermeability of polymersome membranes for many water-soluble compounds is, however, a challenge that has to be overcome if polymersomes are to be used as nanoreactors. To this end, a multitude of approaches have been developed. A prominent example is the reconstitution of membrane proteins in the bio-mimetic polymersome membrane, as this equips the vesicles with highly evolved and specific pores, many of which can be triggered by external stimuli. ${ }^{[5,12]}$ However, membrane proteins are difficult to work with, and are expensive. Simpler approaches are to prepare vesicles with a porous membrane, ${ }^{[13]}$ to induce permeability by light-induced crosslinking reactions between polymers, ${ }^{[14]}$ or to increase the polarity of the hydrophobic leaflet so that it becomes permeable for the desired substrates (vide infra).

Another possibility is to use polymers that are intrinsically permeable for the substrates of a specific enzyme. For example, we were asked to prepare a nanoreactor for laccases. These commercially relevant oxidoreductases are used, e.g. to bleach denim textiles, to cure coatings, or to delignify paper pulp. The aim was to encapsulate the enzyme in order to protect it against degrading and inhibition agents. Laccases are monooxygenases that use oxygen to oxidize substrates such as phenols and aromatic amines. We encapsulated laccases in polymersomes made out of poly(N-vinylpyrrolidone)-block-poly(dimethylsiloxane)-blockpoly(N-vinylpyrrolidone) triblock copolymers (PNVP- $b$-PDMS$b$-PNVP) (Fig. 1). ${ }^{[15]}$ Both types of polymer blocks are highly permeable for oxygen and related species. Indeed, oxygen reached the encapsulated laccase, which formed reactive oxygen species (ROS) in the polymersomes. The ROS diffused out of the nanoreactors and oxidized the substrates. At the same time, the enzyme was stable against deactivation by proteinase $\mathrm{K}$ that was added to the outside of the polymersomes. The polymersome membrane even prevented sodium azide, a potent inhibitor for the enzyme, from reaching the laccase. Thus, tailor-made biocatalytic nanoreactors for laccases were obtained. However, it can be anticipated

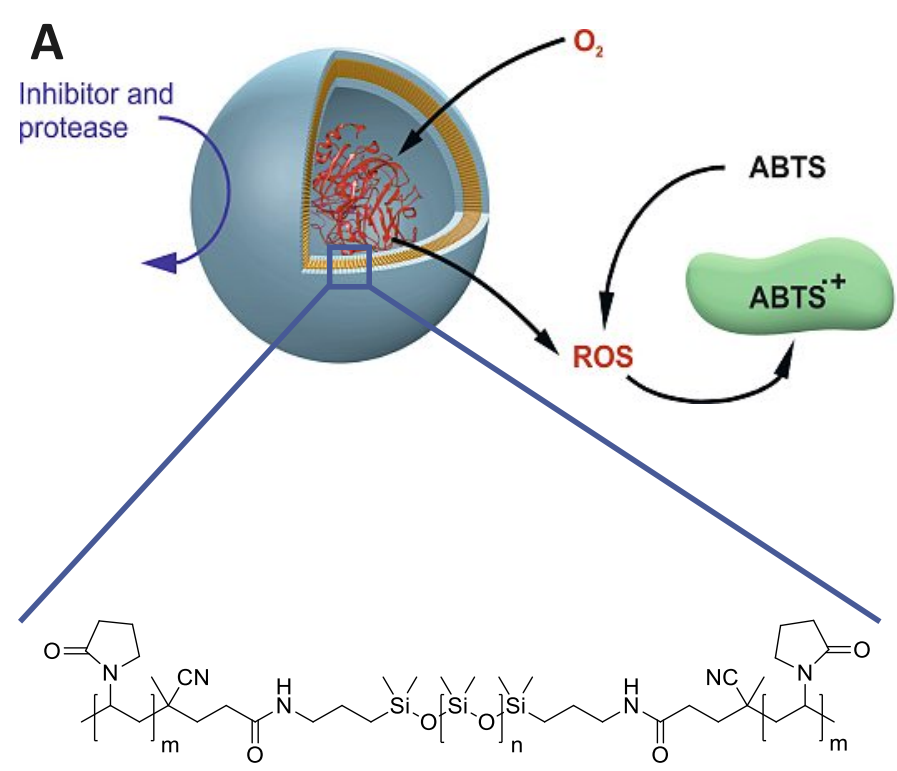

B

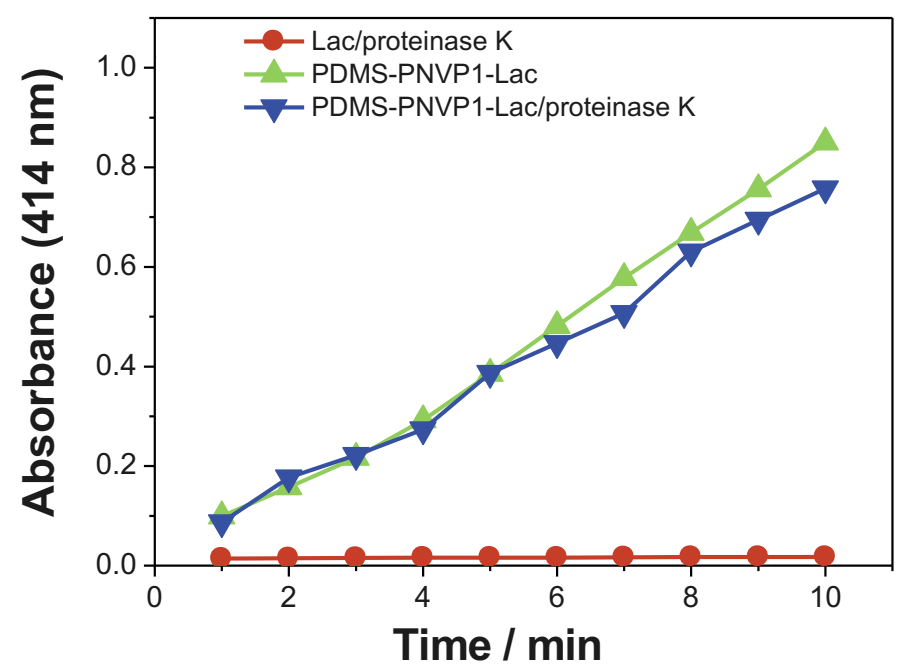

Fig. 1. Polymersome nanoreactors for laccase made out of a block copolymer that is intrinsically permeable for oxygen and reactive oxygen species. A) Schematic depiction; B) ABTS activity assay, showing that the laccase is active within the polymersomes and that the polymersome protects the enzyme from degradation by proteinase K. Figure adapted with permission from ref. [15]. Copyright 2015, American Chemical Society.

that these polymersomes nanoreactors will not be compatible for other types of enzymes because PNVP- $b$-PDMS- $b$-PNVP is not permeable for their substrates.

A generally applicable permeabilization method for the membrane of polymersomes was developed that is not specific to certain types of polymers, and does not depend on the presence of specific functional groups in the polymers. ${ }^{[16]}$ To this end, polymersomes were exposed to the UV-reactive compound 2-hydroxy-4'2-(hydroxyethoxy)-2-methylpropiophenone (Irgacure 2959 or PP$\mathrm{OH}$ ) and the vesicle suspension was irradiated with UV light. The $\alpha$-hydroxyalkylphenone generates radical species that attack the polymers in the membrane and bind to the polymers, increasing their polarity so that the polymersome membrane becomes permeable, as demonstrated in activity measurements with horseradish peroxidase (HRP) encapsulated in the polymersomes (Fig. 2). The photo-permeabilized nanoreactors protected the enzyme against degradation by proteinase K. Moreover, we demonstrated that such photo-permeabilized polymersomes could be filled with hydrophilic polymers by HRP-catalyzed atom transfer radical polymerization (bioATRP). ${ }^{[17]}$ The initiator, reducing agent, and monomer 
could permeate into the polymersomes, where they encountered the enzyme that catalyzed the polymerization. The newly formed polymer chains proved to be too large to cross the polymersome membrane, therefore remained in the polymersomes, as demonstrated by light scattering measurements and by NMR spectroscopy after size exclusion chromatography. Inspiration to produce polymers inside polymersomes is to obtain biomimetic compartments that not only mimic the compartmentalization of cells and organelles, but also feature a dense and viscous interior, which mimics the cytosol of a cell. Such polymer-filled polymersomes could be used to investigate enzyme kinetics in crowded microenvironments.

While the previous examples demonstrate how impermeable, dormant polymersome nanoreactors can be rendered permeable for enzyme substrates, the $\alpha$-hydroxyalkylphenone-based photopermeabilization is a one-time switch-on, and cannot be reverted. However, as stated above, a hallmark of many molecular processes in living organisms is that they are only transiently activated as long as an external stimulus is present. In order to achieve such a response in polymersome nanoreactors, the polymers have to be modified with stimuli-responsive groups that self-revert to their initial state in the absence of the stimulus. If functional groups that switch from a nonpolar to a more polar state are incorporated into the hydrophobic block of the copolymers, they can be used to make the hydrophobic membrane leaflet slightly more hydrophilic and, therefore, permeabilize the polymersomes. Photochromic organic compounds are ideal candidates to create such bio-inspired polymersome nanoreactors, as several photochromes switch their polarity and molecular shape upon light-irradiation. In previous work, we and others have demonstrated that spiropyrans ${ }^{[18,19]}$ and azobenzenes ${ }^{[20]}$ can be used to switch the permeability of polymer membranes. A new type of organic photoswitches that offer a variety of advantages over well-established photochromes were introduced very recently. ${ }^{[21,22]}$ Donor-acceptor Stenhouse adducts (DASAs) switch from a colored open triene-enol isomer to a colorless cyclopentenone isomer upon visible light irradiation (Fig. 3). In the dark, DASAs revert back to their open form within several minutes at room temperature. As the open state is less polar than the closed-ring state, DASAs switch in polarity in response to light. A great advantage of DASAs compared to other photoswitches is that they require visible light to undergo isomerization instead of the much harsher UV light that is needed for tradition- al photoswitches. Therefore, the switching conditions are more compatible with biological systems and potential use in biomedical applications. Moreover, the absorption wavelength of DASAs can be easily tuned in a wide spectral range through the selection of the donor and the acceptor components, allowing preparation of various colored DASAs in a very modular synthesis. A synthetic strategy to functionalize polymers with DASAs was developed, ${ }^{[23]}$ and amphiphilic block copolymers composed of a hydrophilic PEG block and a hydrophobic copolymer of poly(hexyl methacrylate) and DASA-functionalized poly(methacrylamide) were synthesized.[24] The two blocks were self-assembled into polymersomes generating purple and blue DASA polymersomes. Changes in the permeability of their membrane were assessed by the release of a fluorescent dye. Using these polymersomes, lightresponsive biocatalytic nanoreactors were prepared. To this end, HRP was encapsulated into purple DASA polymersomes, while glucose oxidase was encapsulated into blue DASA polymersomes. With the application of white light, purpurogallin was formed at a constant rate showing the successful cascade reaction between the two polymersomes. When irradiated with light of specific wavelengths $(525 \mathrm{~nm}$ or $630 \mathrm{~nm}$ ), the two polymersomes could be switched on selectively. Accordingly, the cascade reaction only worked when both wavelengths were irradiated. Thus, the mixed suspension of the nanoreactors showed a different response depending on the irradiation wavelength, which allowed for the control of individual steps of a reaction cascade in a wavelengthselective way. Importantly, the nanoreactors immediately stopped catalysis in the dark and could be repeatedly activated again by light. Thus, transiently switchable light-responsive polymersomes were obtained. These nanoreactors could be used to orchestrate complex molecular reaction systems, to activate reactions in the focal point of a light source, e.g. within a microfluidic device or in irradiated skin parts in order to be used as drug delivery vehicles.

The concept of switchable polymersomes that feature a small percentage of polarity-switching moieties in their hydrophobic leaflet is of course not limited to light-responsive groups. For example, it can be envisioned to synthesize block copolymers with mechanoresponsive moieties that change their polarity in response to mechanical forces and therefore change the intrinsic permeability of the membrane. Similar, $\mathrm{pH}$ and temperature-responsiveness could be implemented into such polymersomes.
A

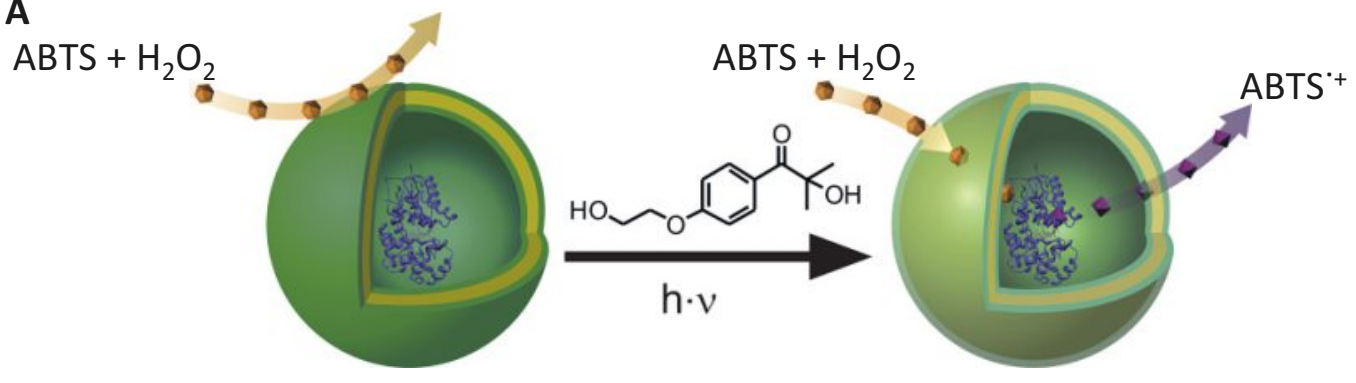

B

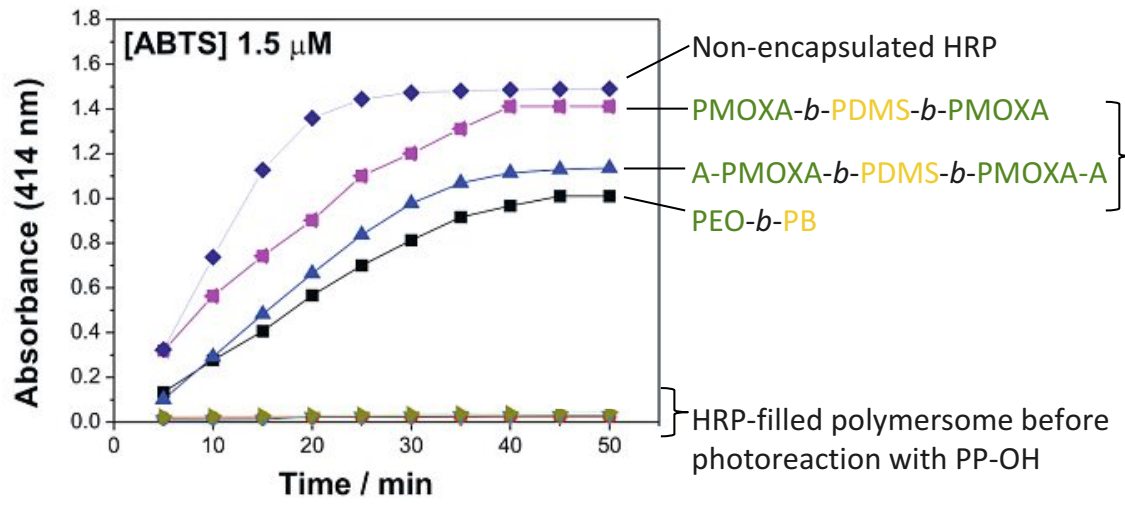

HRP-filled polymersomes after photoreaction with $\mathrm{PP}-\mathrm{OH}$
Fig. 2. Permeabilization of polymersome membranes by photoreaction with the $\alpha$-hydroxyalkylphenone PP-OH as a generally applicable UV-induced method to turn polymersomes into nanoreactors. A) Schematic depiction; B) ABTS activity assay showing that HRP-filled polymersomes only become active when photoreacted with PP-OH. Figure adapted with permission from ref. [16]. Copyright 2013, American Chemical Society. 
A

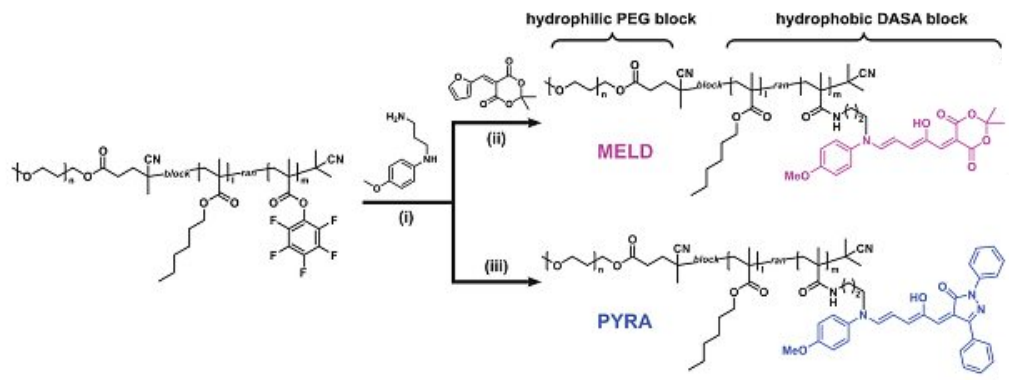

B

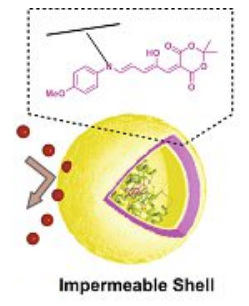

Nanoreactor-Off

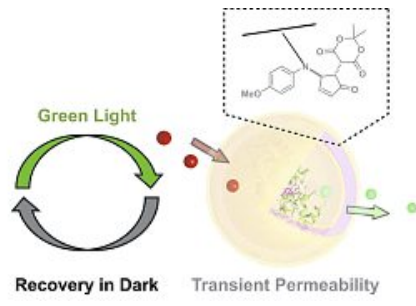

Nanoreactor-On

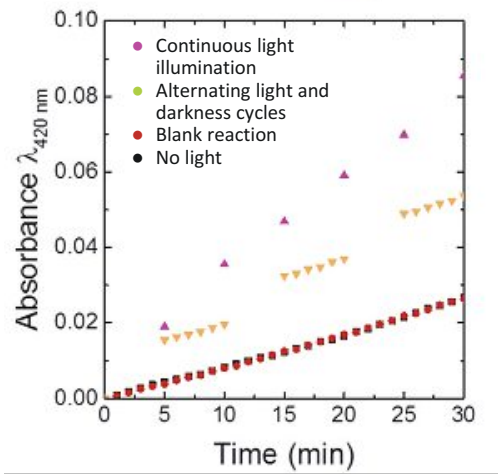

C
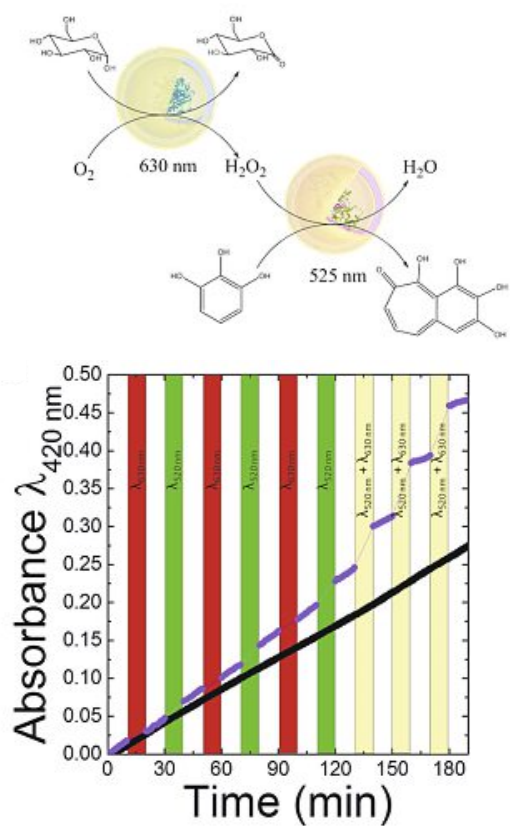

Fig. 3. Wavelength-selective light-responsive DASA-functionalized polymersome nanoreactors that become transiently active when irradiated with visible light. A) Synthesis scheme towards two types of DASA-functionalized block copolymers; B) Catalysis of the reaction of pyrogllol with hydrogen peroxide by HRP in MELD-polymersomes; C) Glucose oxidase - HRP cascade reaction with a mixture of PYRA and MELD-polymersome nanoreactors. Figure adapted with permission from ref. [24]. Copyright 2018, American Chemical Society.

\section{Conclusions}

In conclusion, polymersomes are ideally suited to create bioinspired and stimuli-responsive nanoreactors. Such nanoreactors can be used to protect their catalytic cargo against degradation and to control the reactions that happen inside of the nanoreactors by external stimuli. As such, nanoreactors can be applied to challenging environments, which otherwise would inhibit enzyme activity, e.g. in technical and biomedical applications. The major challenge will be to implement such nanoreactors as part of complex catalytic systems, were multi-step cascade reactions are orchestrated by a variety of different, individually controllable nanoreactors, thereby approaching the complexity of metabolic pathways and feedback mechanisms encountered in living cells.

\section{Acknowledgements}

This work was supported by the Swiss National Science Foundation through the National Center of Competence in Research (NCCR) BioInspired Materials and the project PP00P2_172927. We thank Dr. Kyle J. Rodriguez for editing the manuscript. Foto of NB: Copyright Christopher Schaller.

Received: November 18, 2018

[1] M. Marguet, C. Bonduelle, S. Lecommandoux, Chem. Soc. Rev. 2013, 42, 512.

[2] R. Merindol, A. Walther, Chem. Soc. Rev. 2017, 46, 5588.

[3] K. Renggli, P. Baumann, K. Langowska, O. Onaca, N. Bruns, W. Meier, Adv. Funct. Mater. 2011, 21, 1241.

[4] R. J. R. W. Peters, I. Louzao, J. C. M. van Hest, Chem. Sci. 2012, 3, 335

[5] C. G. Palivan, R. Goers, A. Najer, X. Zhang, A. Car, W. Meier, Chem. Soc. Rev. 2016, 45, 377.

[6] J. Gaitzsch, X. Huang, B. Voit, Chem. Rev. (Washington, DC, U. S.) 2016, 116,1053
[7] E. Rideau, R. Dimova, P. Schwille, F. R. Wurm, K. Landfester, Chem. Soc. Rev. 2018, 47, 8572.

[8] M. Rother, M. G. Nussbaumer, K. Renggli, N. Bruns, Chem. Soc. Rev. 2016, $45,6213$.

[9] W. M. Aumiller, M. Uchida, T. Douglas, Chem. Soc. Rev. 2018, 47, 3433.

[10] P. Cotanda, N. Petzetakis, R. K. O'Reilly, MRS Commun. 2012, 2, 119.

[11] F. Itel, M. Chami, A. Najer, S. Lörcher, D. Wu, I. A. Dinu, W. Meier, Macromolecules 2014, 47, 7588.

[12] W. Meier, C. Nardin, M. Winterhalter, Angew. Chem., Int. Ed. 2000, 39, 4599.

[13] S. M. Kuiper, M. Nallani, D. M. Vriezema, J. J. L. M. Cornelissen, J. C. M. van Hest, R. J. M. Nolte, A. E. Rowan, Org. Biomol. Chem. 2008, 6, 4315.

[14] J. Gaitzsch, D. Appelhans, L. Wang, G. Battaglia, B. Voit, Angew. Chem., Int. Ed. 2012, 51, 4448.

[15] M. Spulber, P. Baumann, S. S. Saxer, U. Pieles, W. Meier, N. Bruns, Biomacromolecules 2014, 15, 1469.

[16] M. Spulber, A. Najer, K. Winkelbach, O. Glaied, M. Waser, U. Pieles, M. Meier, N. Bruns, J. Am. Chem. Soc. 2013, 135, 9204.

[17] M. V. Dinu, M. Spulber, K. Renggli, D. Wu, C. A. Monnier, A. Petri-Fink, N. Bruns, Macromol. Rapid Commun. 2015, 36, 507.

[18] K. Schöller, S. Küpfer, L. Baumann, P. M. Hoyer, D. de Courten, R. M. Rossi, A. Vetushka, M. Wolf, N. Bruns, L. J. Scherer, Adv. Funct. Mater. 2014, 24, 5194.

[19] X. Wang, J. Hu, G. Liu, J. Tian, H. Wang, M. Gong, S. Liu, J. Amer. Chem. Soc. 2015, 137, 15262.

[20] E. Blasco, J. L. Serrano, M. Piñol, L. Oriol, Macromolecules 2013, 46, 5951.

[21] J. R. Hemmer, S. O. Poelma, N. Treat, Z. A. Page, N. D. Dolinski, Y. J. Diaz, W. Tomlinson, K. D. Clark, J. P. Hooper, C. Hawker, J. Read de Alaniz, J. Am. Chem. Soc. 2016, 138, 13960.

[22] M. M. Lerch, W. Szymanski, B. L. Feringa, Chem. Soc. Rev. 2018, 47, 1910.

[23] S. Ulrich, J. R. Hemmer, Z. A. Page, N. D. Dolinski, O. Rifaie-Graham, N. Bruns, C. J. Hawker, L. F. Boesel, J. Read de Alaniz, ACS Macro Lett. 2017, 6,738

[24] O. Rifaie-Graham, S. Ulrich, N. F. B. Galensowske, S. Balog, M. Chami, D. Rentsch, J. R. Hemmer, J. Read de Alaniz, L. F. Boesel, N. Bruns, J. Am. Chem. Soc. 2018, 140, 8027. 